\title{
Monte Carlo Simulation of Missile Trajectories DISPERSION DUE TO IMPERFECTLY MANUFACTURED WARHEAD
}

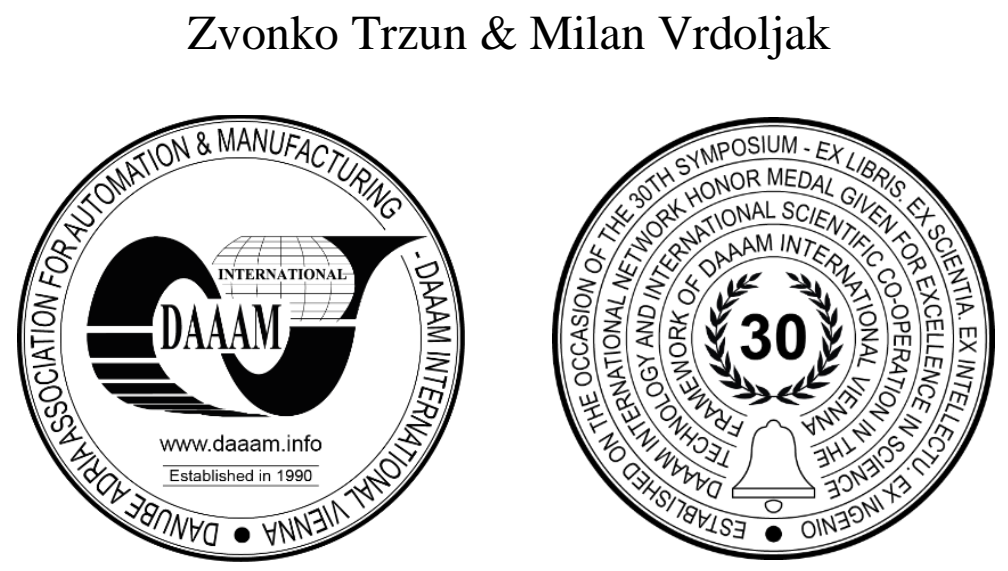

This Publication has to be referred as: Trzun, Z[vonko] \& Vrdoljak, M[ilan] (2020). Monte Carlo Simulation of Missile Trajectories Dispersion due to Imperfectly Manufactured Warhead, Proceedings of the 31st DAAAM International Symposium, pp.0574-0583, B. Katalinic (Ed.), Published by DAAAM International, ISBN 978-3-90273429-7, ISSN 1726-9679, Vienna, Austria

DOI: $10.2507 / 31$ st.daaam.proceedings.079

\begin{abstract}
The missile accuracy strongly depends on the quality of production. It is necessary to find the right optimum between the criterion of cost and the criterion of precision. In this paper, the warhead manufacturing error is simulated using the Monte Carlo method, treated as nondeterministic disturbance, and dispersed according to suitable axisymmetric distribution. A link is proposed between the missile 3D CAD model, a 6DOF model of its flight, and the statistical analysis of trajectories dispersion parameters. The presented method is an excerpt from a broader analysis that reveals statistically significant differences between the consequences of individual errors, especially their impact on missile accuracy. By imposing especially strict tolerances in the critical stages of production, it is possible to significantly improve the efficiency of the missile, with a minimal increase in the final product cost.
\end{abstract}

Keywords: missile dispersion; 3D CAD modelling; 6DOF; Monte Carlo simulation; quality optimization

\section{Introduction}

Manufacturing errors are always present in the production process. Since in military production any imprecision could result in tragic consequences and even loss of human lives [1], great attention is paid to raising the quality of military production. On the other hand, in today's engineering production there is strong pressure on productivity and economy. Strict requirements make every aspect of the entire process important, with the goal to manufacture parts of sufficient quality at reasonable costs [2]. The purpose of the study is to find the right balance between the requirements for the high projectile's precision and the requirement for its low production cost. Therefore, the questions must be resolved which production errors contribute more, and which less to the overall missile impact points dispersion. Lower quality of production can be tolerated for less sensitive steps, while the strictest production tolerances should be imposed on steps identified as critical.

\section{Related Work}

Important parameters should be closely inspected inside the interval of possible variations, to identify potential problems [3], [4]. As for the variation of projectile's characteristics, production errors are the main generator for characteristics inconsistency even if not specifically mentioned [5]. 
Authors generally focus on groups of critical parameters and analyse their alteration within set limits. The problem is that these limits are sometimes arbitrarily established or differ significantly for other reasons. In most cases though, earlier works introduce assumptions that decrease the general applicability:

- disturbances of the missile geometric and inertia characteristics are not related to the production phase;

- $\quad$-missiles are assumed to be axisymmetric (transverse moments of inertia are same Iy $=\mathrm{Iz}$ );

- the centre of gravity remains on the $\mathrm{x}$-axis.

Of particular interest is the paper [6] where authors develop a 6DOF model of a dynamically unbalanced projectile. However, this work treats only classical artillery projectiles (but not missiles) and does not mention the thrust force asymmetry. The 6DOF flight model is developed in the aeroballistic coordinate system used for artillery shells, but very rarely for missiles. Mentioned papers are a valuable source providing the general method of how to analyse the variations of missile characteristics, and this problem is further developed in our work.

\section{Research Description and Proposed Novelties}

As described in [7], authors propose a method that connects errors made during the production of a missile with the resulting dispersion of trajectories and position of impact points. Powerful 3D CAD software packages have opened up new perspectives for a number of different techniques (FEM, CAM, off-line programming of industrial robots, etc.), and the potential of 3D CAD model's use has been recognized in processes of modelling and simulation of dynamic systems [8]. Our method also combines a parametrically-defined 3D model of a well-known rocket, with the improved $6 \mathrm{DOF}$ model of flight, adjusted so to facilitate tracking of the projectile with geometrical and mass asymmetry.

The 3D model, as shown in Figure 1, is created to reflect the characteristics of an ideal rocket. The occurrence of manufacturing error is then simulated, after which the CAD software delivers new inertia characteristics for the "real" missile (differing from the ideal rocket). Data gathered in this way becomes the input for the improved 6DOF model of flight. The final displacement of impact point $\delta \vec{D}$, following from the 6DOF flight model, can be expressed using the finite difference method:

$$
\delta \vec{D}=\frac{\partial \vec{D}}{\partial p_{1}} \delta p_{1}+\frac{\partial \vec{D}}{\partial p_{2}} \delta p_{2}+\ldots \frac{\partial \vec{D}}{\partial p_{n}} \delta p_{n}=\sum_{i=1}^{n} \frac{\partial \vec{D}}{\partial p_{i}} \delta p_{i}
$$

where $\delta p_{i}=$ variation of parameter, and $\partial \vec{D} / \partial p_{i}=$ sensitivity of impact point position due to particular manufacturing error. Applied to our work, variation of parameter $\delta p_{i}$ represents disturbances introduced into the 3D CAD model.

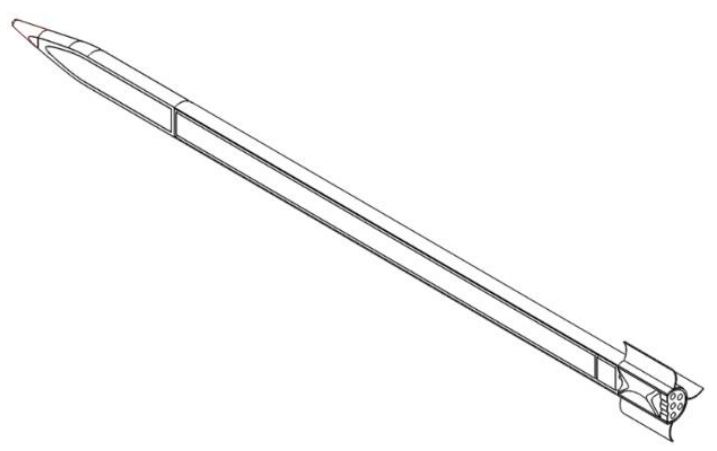

Fig. 1. The cross-section of a 3D CAD model of the rocket

The differential coefficient $\partial \vec{D} / \partial p_{i}$ comes from the 6DOF model of missile flight. As for the 6DOF, we use the adjusted model that differs from the classical one to facilitate tracking of the dynamically unbalanced missile. It is developed in the geometrical coordinate system (linked to axes of external surface symmetry), while classical 6DOF [9] is developed in the frame coordinate system, or its non-rotating version the aeroballistics coordinate system. Furthermore, although the governing equations are the same as for the classical 6DOF model:

$$
\begin{aligned}
& m\left(\dot{\mathbf{V}}_{K}^{G}+\tilde{\mathbf{\Omega}}_{G} \cdot \mathbf{V}_{K}^{G}\right)=\mathbf{F}_{A}^{G}+\mathbf{F}_{T}^{G}+\mathbf{L}_{G O} m \mathbf{g}^{O}-m \mathbf{a}_{c o r}^{G} \\
& \dot{\mathbf{H}}^{G}+\tilde{\boldsymbol{\Omega}}_{G} \cdot \mathbf{H}^{G}=\mathbf{M}_{A}^{G}+\mathbf{M}_{T}^{G} \\
& V_{k x}^{O}=\dot{\varphi}_{E}(R+h)
\end{aligned}
$$




$$
\begin{aligned}
& V_{k y}^{O}=\left(\dot{\lambda}+\Omega_{E}\right) \cdot(R+h) \cos \varphi_{E} \\
& V_{k z}^{O}=-\dot{h} \\
& \dot{\mathbf{s}}^{G}=\mathbf{R}^{-1}(\varphi, \vartheta) \cdot\left(\mathbf{\Omega}_{G}^{G}-\mathbf{\Omega}_{O}^{G}\right) \\
& \mathbf{R}=\left[\begin{array}{ccc}
1 & 0 & -\sin \theta_{G} \\
0 & \cos \varphi_{G} & \sin \varphi_{G} \cos \theta_{G} \\
0 & -\sin \varphi_{G} & \cos \varphi_{G} \cos \theta_{G}
\end{array}\right]
\end{aligned}
$$

there are also differences since in proposed model many assumptions (otherwise present in the classical 6DOF) do not exist anymore. For instance, not only does inertia tensor looks different, with members outside the main diagonal being not equal to zero $\left(I_{x y} \neq I_{y z} \neq I_{z x} \neq 0\right)$, but also transverse moments of inertia do not have to be the same $\left(I_{y} \neq I_{z}\right)$. The vector of state now incorporates components of angular momentum, and not angular velocity as in classical 6DOF:

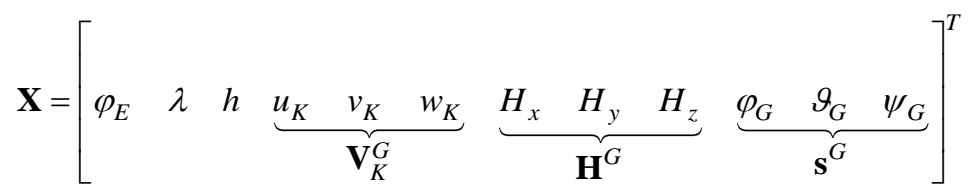

The superscript "G" points the variable is expressed in the geometrical coordinate system (G-CS).

\section{Monte - Carlo Simulation}

The impact of production errors can be examined in two ways:

- by treating errors as deterministic variables and finding a functional relation between particular error and the resulting deviation of the impact point position;

- by treating errors as nondeterministic variables, using simulations, and statistically processing the aggregated simulation outcomes.

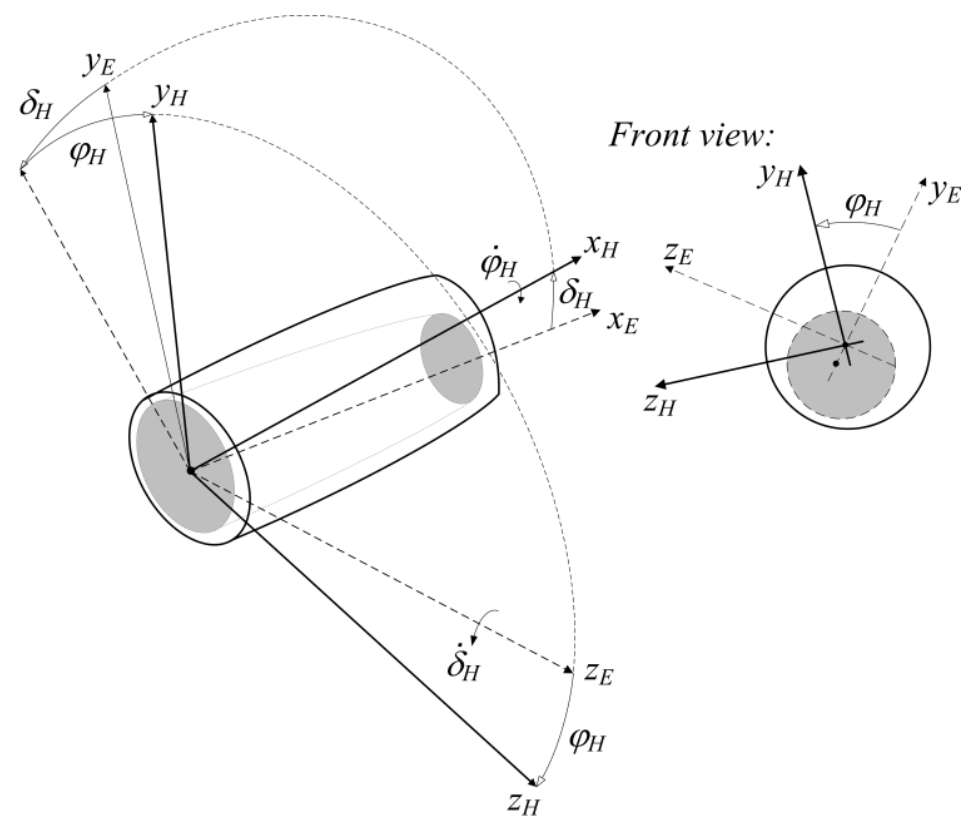

Fig. 2. The erroneously manufactured warhead

The first method is presented in [7], and therefore is only briefly commented on for comparison with the second method. As a case study, a warhead manufacturing error is selected, where the axes of symmetry of the inner surface do not coincide with the axes of symmetry of the outer surface. The error is defined by two angles:

- by the angle $\delta_{H}$ which gives the rotation of the inner surface axis of symmetry $x_{E}$ relative to the outer surface axis of symmetry $x_{H}$, in the plane $x_{E}-y_{E}$;

- by the angle $\varphi_{H}$ which gives the radial rotation of that plane relative to the reference plane $x_{H}-y_{H}$, as in Figure 2 . 
If the mentioned error is analysed so that the angles $\delta_{H}$ and $\varphi_{H}$ are treated as deterministic variables, e.g. by fixing the angle $\delta_{H}$ at a certain value while the radial angle $\varphi_{H}$ is changing within the interval $\varphi_{H}=0-360^{\circ}$, then a curve connecting the missile impact points (usually of elliptical shape) is obtained as shown in Figure 3.

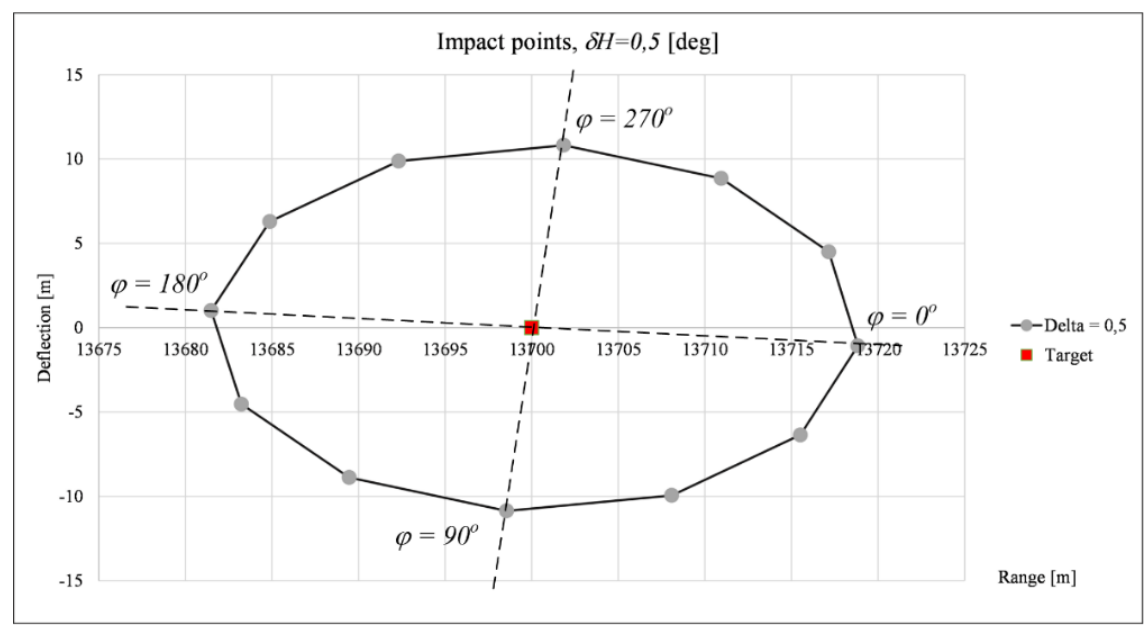

Fig. 3. Impact points for a fixed angle $\delta_{H}$

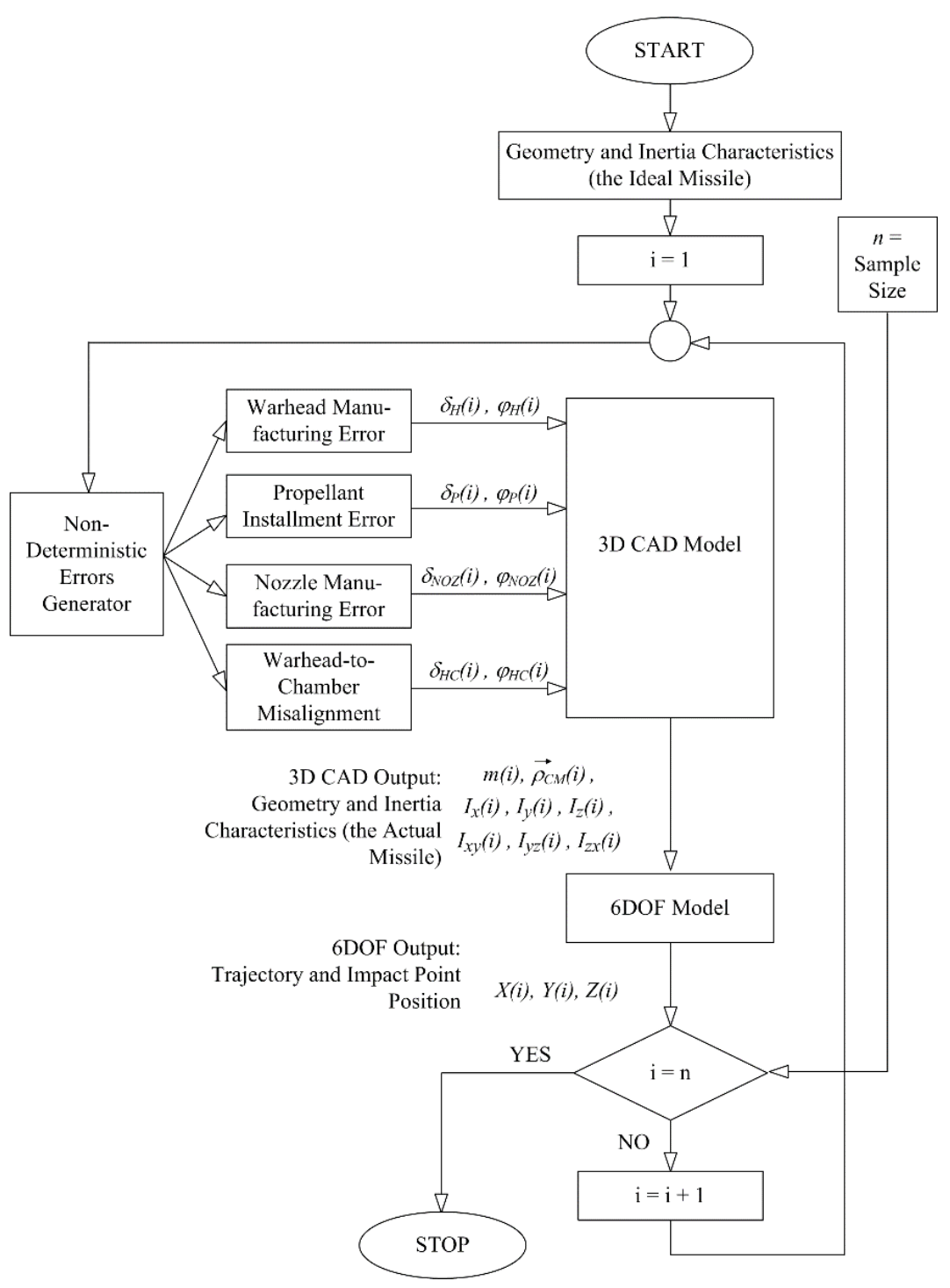

Fig. 4. The algorithm of Monte Carlo simulation 
To analyse the correlation between multiple simultaneously occurring disturbances and the resulting impact point displacement, the most practical method is to use simulation. The most often used is the Monte Carlo simulation whose algorithm is shown in Figure 4. The Monte Carlo simulation relies on repeated nondeterministic sampling to obtain numerical results, with the idea to use the stochastic nature of a sample to ultimately describe processes that may be deterministic in nature. Due to practical limitations, simulation should include only parameters having a particularly strong influence on the missile trajectory. These critical parameters are:

- for the missile - warhead mass, missile aerodynamic parameters, direction and intensity of the thrust force;

- for initial conditions - position and orientation of the launcher; direction and intensity of initial speed, and the transverse angular velocity;

- for the atmosphere: temperature, pressure, humidity, and especially the wind.

To single out only the impact of manufacturing errors, it is here assumed that there are no other disturbances. In this way, it is possible to analyse the impact of production quality without the interference of other factors that could correlate with analysed errors. It is also possible (and actually highly useful) to analyse the impact of only one error at a time, identifying in this way the most critical errors. In our broader work, we examined the effect that various manufacturing errors have on the missile trajectory and the dispersion of impact points, but in this paper only the effect of warhead manufacturing error is analysed.

\subsection{Simulation Parameters}

When designing a simulation, the required sample size (number of iterations) should be determined so that the results can be accepted with the required confidence. This step is sometimes skipped and the sample size is simply taken empirically: the research [10] states that in only $9 \%$ of cases, the authors of studies explain how they choose a specific sample size. In our paper, the sample size is selected based on the central limit theorem.

If the realizations within the random samples $X_{1}, X_{2}, \ldots, X_{n}$ are dispersed according to probability distribution $F$, with mean $a$ and variance $\sigma^{2}$, then (provided that $n$ is large enough) the mean values of samples $\bar{X}$ are approximately dispersed according to the normal distribution, with parameters $a_{\bar{X}}=a$ and $\sigma_{\bar{X}}{ }^{2}=\sigma^{2} / n$. This approximation is better when the sample size $n$ increases.

The error of the Monte Carlo simulation is $\sigma / \sqrt{n}$, meaning it can be reduced either by increasing the number of simulations $n$, or by reducing the variance $\sigma^{2}$. An increase in sample size is a more common option, since variance reducing methods introduce new assumptions which generally do not have to be met. The required number of iterations $n$ can be linked to the confidence interval of mean estimation, or the confidence interval of standard deviation estimation, both for the given sample. To the test (i.e. to the sample $x_{1}, x_{2}, \ldots x_{n}$ ) a random variable is linked

$$
T=\frac{\bar{X}-a}{S} \sqrt{n}
$$

where $S$ is the random variable

$$
S=\sqrt{\frac{\sum_{i=1}^{n}\left(X_{i}-\bar{X}\right)^{2}}{n-1}}
$$

and $T$ follows the Student distribution

$$
f(t)=\frac{B_{n}}{\sqrt{\left(1+\frac{t^{2}}{n-1}\right)^{n}}}
$$

If the probability that $\mathrm{T}$ is inside a given interval $\left(-t_{p}, t_{p}\right)$ is

$$
p\left(T<t_{p}\right)=\int_{-t_{p}}^{+t_{p}} f(t, n) d t
$$

then it can be proved that under desired probability $p$, the mean of a sample is inside the confidence interval 


$$
\bar{X}-t_{p} \frac{S}{\sqrt{n}}<a<\bar{X}+t_{p} \frac{S}{\sqrt{n}}
$$

The half-width of the confidence interval around the average value $\bar{X}$ can be expressed as

$$
\Delta=t_{p} \frac{S}{\sqrt{n}}
$$

from which a simple transformation yields the required number of iterations $n$ :

$$
n=\left(t_{p} \frac{S}{\Delta}\right)^{2}
$$

The number $n$ cannot be predetermined according to the above expressions, because the simulation has yet to be performed and there is no data for $\bar{X}$ or $S$. Additionally, a parameter $t_{p}$ depends on the selected confidence level $p$, and also on the degrees of freedom number $D F=n-1$ that is yet to be calculated. Therefore, $n$ must be determined iteratively.

It is convenient to set $\Delta$ as a relative value in relation to $S$. Since the dispersion of impact points is usually analysed separately in range (with standard deviation $S_{X}$ ) and separately in deflection (parameter $S_{Z}$ ), in this study the ratio $\Delta / S_{X}=0.2$ is chosen, giving $n=99$. For this simulation, a larger sample $(n=300)$ is chosen to obtain an even narrower confidence interval.

\subsection{Input Data Dispersion}

The input data are dispersed according to the distribution that best matches empirical data and literature. The occurrence of the warhead manufacturing error is simulated. No other disturbances are present. The error of warhead is simulated via the angles $\delta_{H}$ and $\varphi_{H}$. The planes in which lies the $\delta_{H}$ angle are dispersed symmetrically around the $x_{H}$ reference axis. This means that the radial angle $\varphi_{H}$ is dispersed according to the uniform distribution, within the interval $\varphi_{H}=0-360^{\circ}$.

As for the $\delta_{H}$ angle, it is always positive. There are several suitable distribution functions to describe such dispersion, for example Rayleigh distribution, Half-normal, Log-normal, etc. For this paper, a dispersion according to Rayleigh distribution is assumed. The Rayleigh distribution is a special case of the Weibull distribution, of a general form

$$
f\left(\delta_{H}\right)= \begin{cases}\frac{\delta_{H}}{\sigma^{2}} e^{-\left(\frac{\delta_{H}{ }^{2}}{2 \sigma^{2}}\right)} & \text { za } \delta_{H} \geq 0 \\ 0 & \text { za } \delta_{H}<0\end{cases}
$$

where $\sigma=$ scale parameter. The form of distribution is graphically shown in Figure 5 .

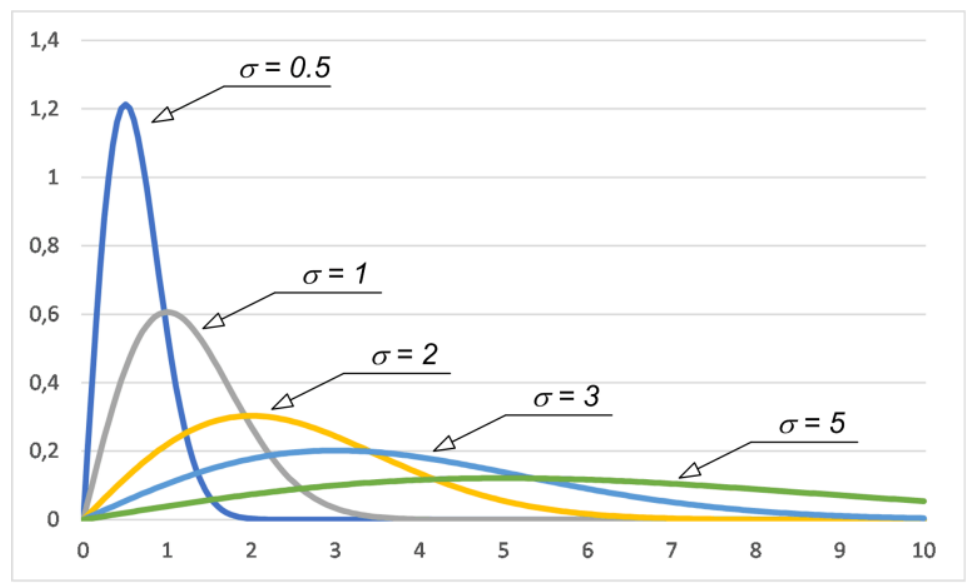

Fig. 5. Rayleigh distribution for different scale parameters $\sigma$ 
The maximum probability density is at $\delta_{m}$, found from the derivation of the density function $f^{\prime}\left(\delta_{m}\right)=0$ :

$f^{\prime}\left(\delta_{m}\right)=-\frac{\left(\delta_{m}^{2}-\sigma^{2}\right)}{\sigma^{2}} \exp \left(-\frac{\delta_{m}^{2}}{2 \sigma^{2}}\right)=0 \quad \rightarrow \quad \delta_{m}=\sigma$

after which (15) becomes

$$
f\left(\delta_{H}\right)=\frac{\delta_{H}}{\delta_{m}^{2}} \exp \left(-\frac{\delta_{H}{ }^{2}}{2 \delta_{m}^{2}}\right)
$$

and the Rayleigh distribution becomes one-parameter, depending only on $\delta_{m}$. Unlike the normal distribution, mean is not at the maximum probability density but at $a$ (linearly correlated with $\delta_{m}$ )

$a=\int_{0}^{\infty} \delta_{H} f\left(\delta_{H}\right) d \delta_{H}=\delta_{m} \sqrt{\frac{\pi}{2}}$

and the median is at $\delta_{0}$, also dependent only on $\delta_{m}$ parameter:

$\delta_{0}=\delta_{m} \sqrt{2 \ln 2}=1,177 \cdot \delta_{m}$

The parameter $\delta_{m}$ for our simulation is chosen so that there is $p=0.995$ probability that $\delta_{H}<0,5^{\circ}$ (which is the maximum allowed angle of error):

$$
\begin{gathered}
p\left(\delta<\delta_{\max }\right)=1-\exp \left(-\frac{\delta_{\max }^{2}}{2 \delta_{m}^{2}}\right)=0.995 \\
\rightarrow \text { if } \delta_{\max }=0.5^{\circ}, \text { then } \delta_{m}=0.15^{\circ}
\end{gathered}
$$

\section{Results and Analysis}

The launching of 300 missiles was simulated. Conditions assumed to be the same for all 300 missiles are:

- angle of elevation and angle of azimuth;

- standard ICAO atmosphere;

- target position in the local coordinate system: $\boldsymbol{\rho}_{C}^{L}=[13700,0,0]$.

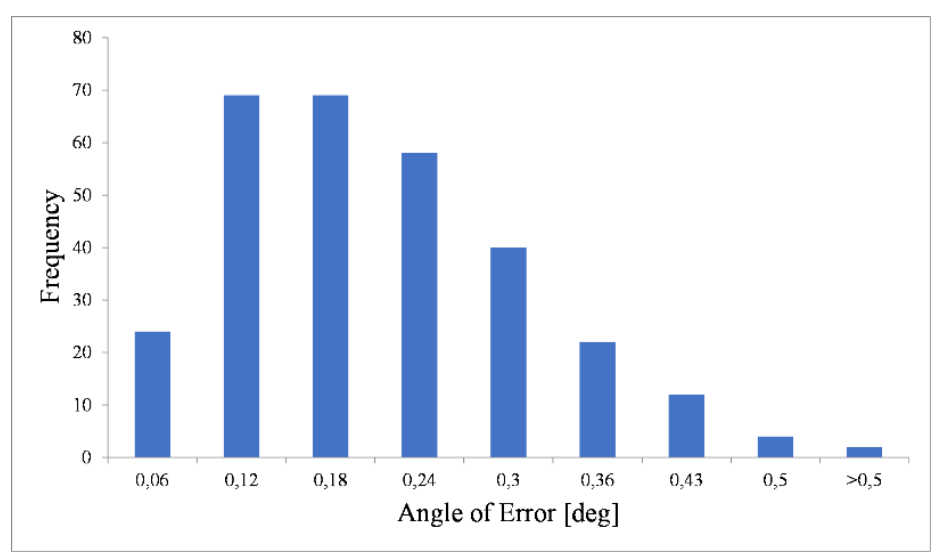

Fig. 6. Frequencies of the warhead error angle $\delta_{H}$

Conditions simulated to be different for each individual rocket are:

- angle of error $\delta_{H}$;

- radial angle $\varphi_{H}$;

- inertia characteristics, changing due to manufacturing error: 
○ centre of gravity position $\boldsymbol{\rho}_{m}$;

$\circ \quad$ inertia tensor $\mathbf{I}^{G}$;

$\circ$ principal axes direction.

The angle $\varphi_{H}$ is dispersed uniformly about the $x_{H}$ axis, and the angle $\delta_{H}$ is dispersed according to the Rayleigh distribution with the scale parameter $\delta_{m}=0.15^{\circ}$. The process of production is simulated, accompanied by the occurrence of production errors. The quality control is not simulated - i.e. a few missiles where $\delta_{H}>0.5^{\circ}$ are also included in simulation, to analyse their effect on missile inaccuracy. Figure 6 shows the frequencies of $\delta_{H}$ angle. A characteristic form of Rayleigh distribution is recognized. Of the 300 simulated $\delta_{H}$ angles, two are greater than 0.5 . Such projectiles would be rejected during the quality control (percentage of discarded products: $0.67 \%$ of the total production, which is quite acceptable). The Figure 7 gives the impact points dispersion around the target (marked as a red square):

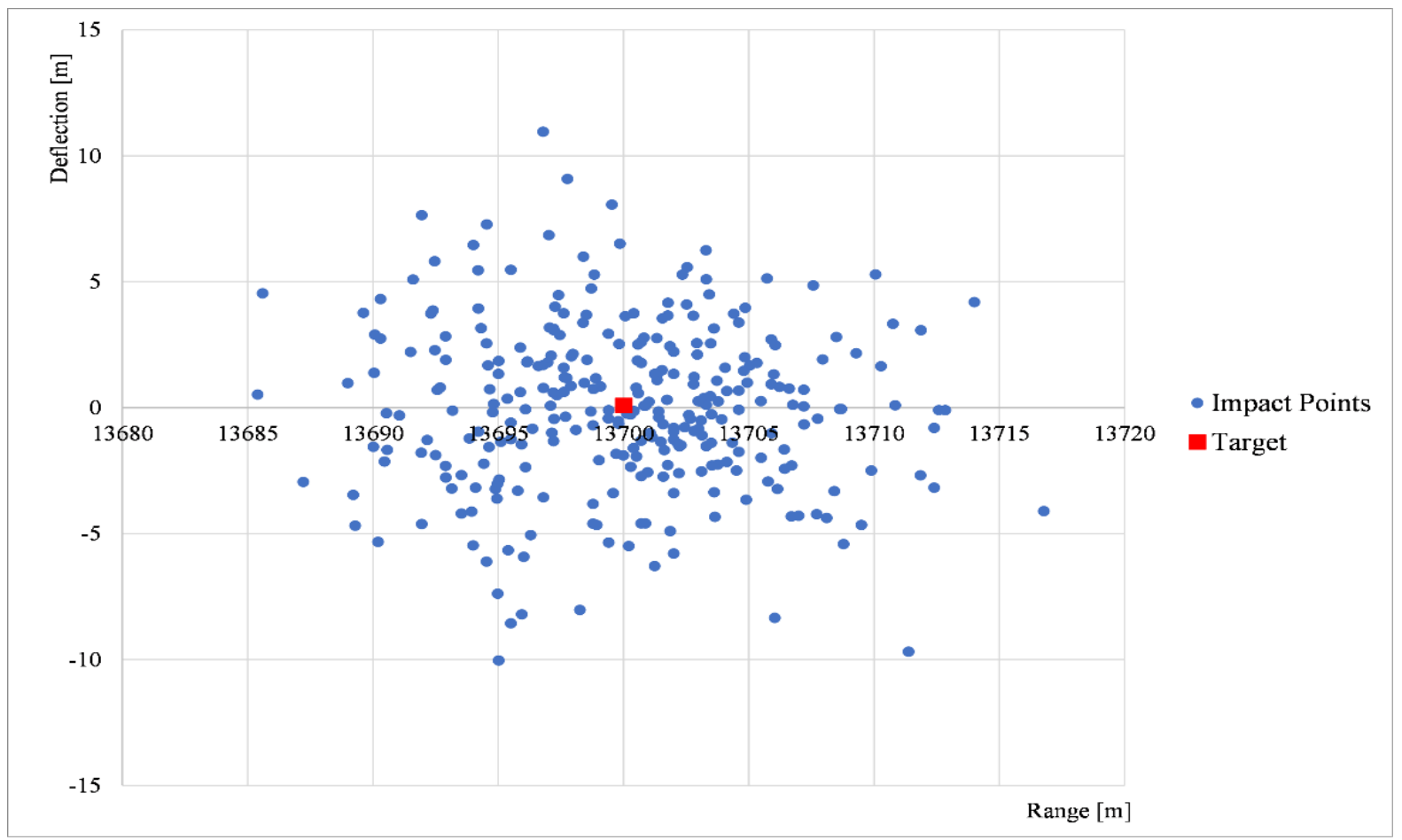

Fig. 7. Dispersion of impact points due to the warhead manufacturing error

The impact points dispersion shows a strong concentrating around the position of the target, and a decrease in frequency as the distance from the target grows. Artillery theory assumes that the impact points are dispersed according to the normal distribution. This assumption should be verified. The first way to assess the normality of the sample is a visual test, by reviewing histograms that give frequencies of the impact points position in range and in deflection.

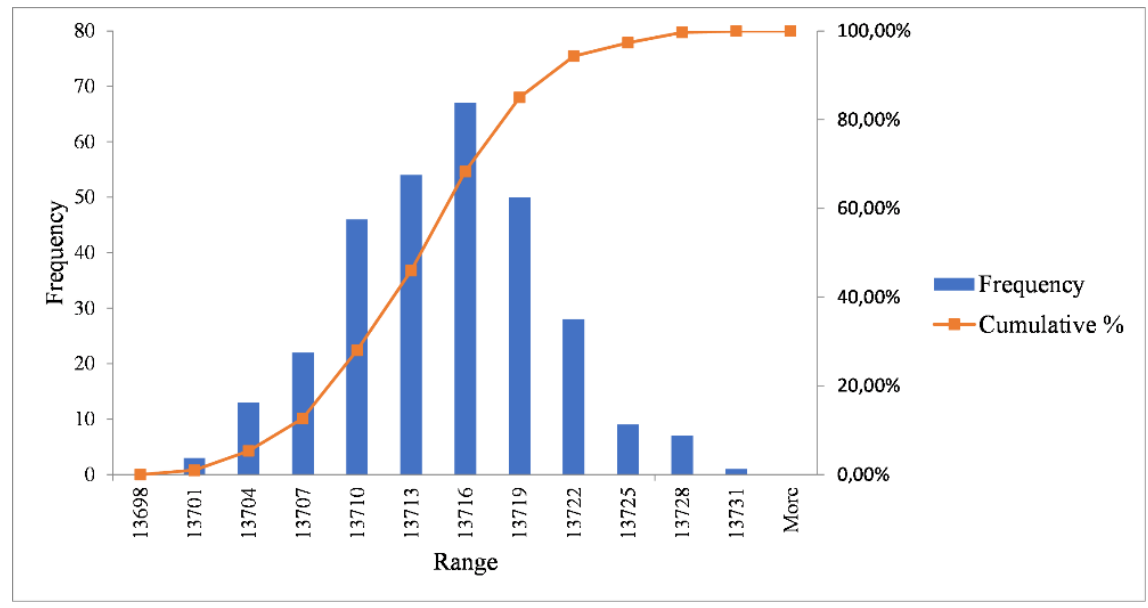




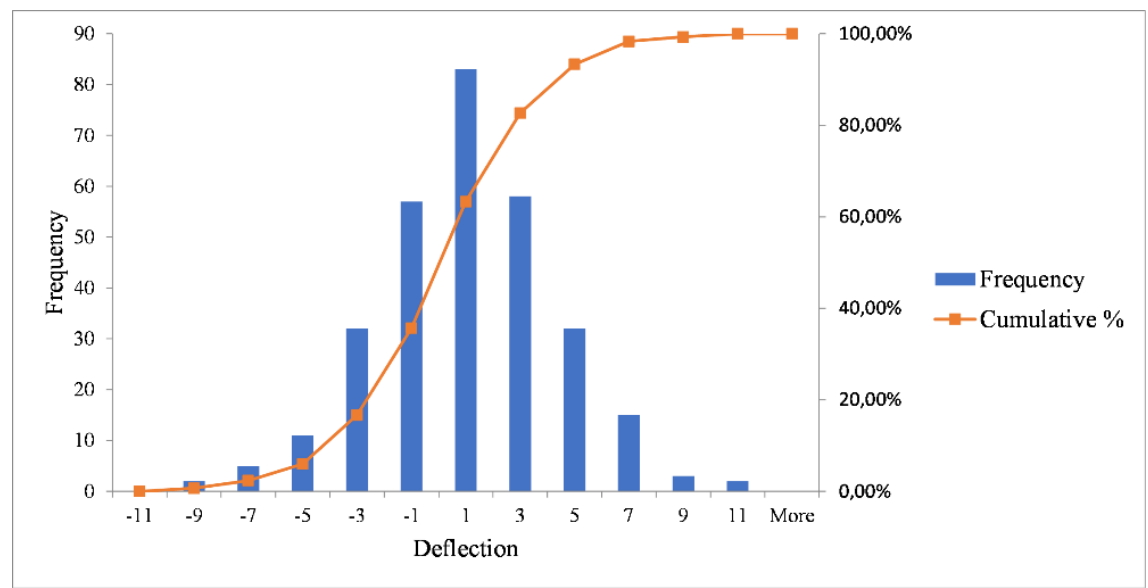

Fig. 8. Frequencies of impact points positions, in range (up) and in deflection (down)

Histograms shown in Figure 8 give a strong indication the output sample (impact points) is normally distributed. To additionally back up the graphical method, the verification of the sample normality can be performed by various statistical tests: Kolmogorov-Smirnov test, Lilliefors version of the Kolmogorov-Smirnov test, Shapiro-Wilk test, etc. In this paper a Kolmogorov-Smirnov test is chosen, defined as follows:

1. $H_{0}$ (null hypothesis): data follow a certain distribution

2. $H_{a}$ (alternative hypothesis): data do not follow a specific distribution

3. $\alpha$ - degree of significance

4. critical values - the hypothesis related to the type of distribution is rejected if the test parameter $D$ is greater than the critical value

5. Test parameter $D$ is defined as the largest vertical difference between the cumulative function of the normal probability distribution $F_{0}(x)$ and the empirical distribution function $F_{n}(x)$.

For a specific sample of 300 simulated missile launches, a very low critical value $D=0.035$ and a high estimated probability $p=83.98 \%$ were calculated. This suggests that this is indeed a normally distributed sample. The estimation of the standard deviation for range is $S_{X}=5.5 \mathrm{~m}$, while the mean is estimated inside the confidence interval $a_{X}=13700 \pm 0.6 m$ with a $95 \%$ probability.

In analogy, the estimate of the standard deviation for deflection is $S_{Z}=3.3 \mathrm{~m}$, while the mean is estimated inside the confidence interval $a_{Z}=0 \pm 0.4 m$ (also with a $95 \%$ probability). Results show that parameters of the impact point dispersion can be estimated within a very narrow confidence interval, and with a high confidence. Box plots (shown in Figure 9) graphically depict distribution of impact points through their quartiles, both in range and in deflection. A narrow interquartile range (IQR) is visible, with a small number of outliers.
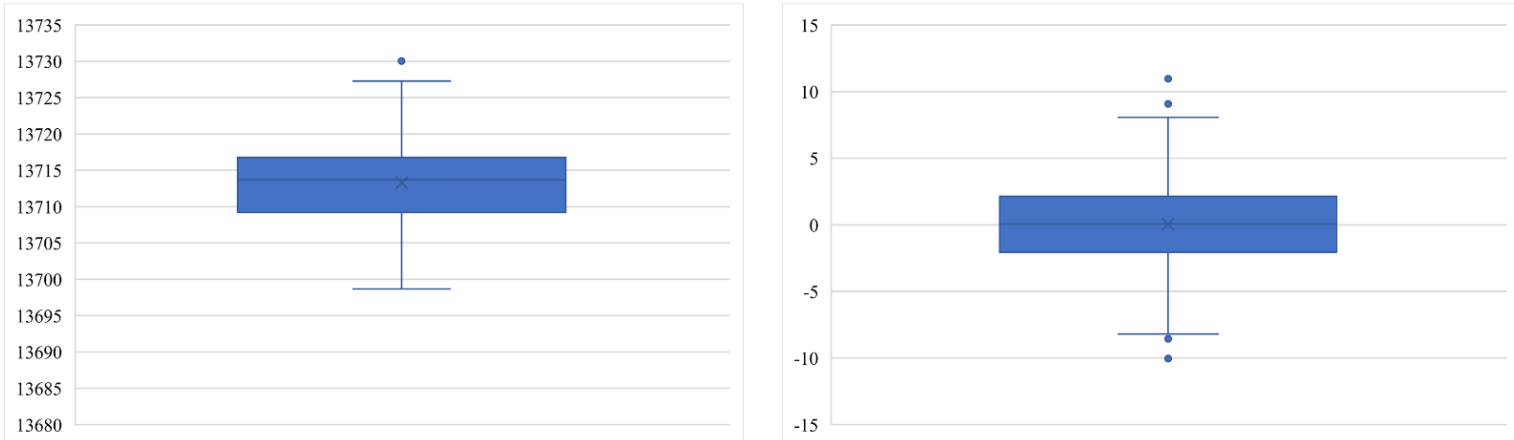

Fig. 9. Box plots showing distribution of impact points position in range (left) and in deflection (right)

Outliers are caused by large angles of error $\delta_{H}$, and the more detailed analysis is left for later work.

\section{Conclusion}

As a contribution to the method of finding the right balance between the requirements for the high projectile's precision and the requirement for its low production cost, a statistical analysis of the missile impact point dispersion due to a single disturbance (warhead manufacturing error) is presented. 
The error is simulated by introducing it into the accurate 3D CAD missile model, after which the CAD software gives the modified inertia characteristics which are then entered into the adjusted 6DOF flight model (described in [7]). The Monte Carlo simulation proves the erroneously manufactured warhead does not contribute significantly to the missile impact points dispersion, and therefore looser tolerances could be recommended for this stage of production.

A described simulation is even more valuable if a large number of disturbances (production errors) occur simultaneously, as they will during the real production. In the future work, each particular error will be analysed (and their correlations as well), to single out the ones that contribute the most to the missile imprecision. Imposing the especially tight tolerances only on these steps of production will improve the missile precision, while still maintaining the cost of the final product as low as possible.

\section{Literature}

[1] Brady, R. \& Goethals, P. (2019). A comparative analysis of contemporary $155 \mathrm{~mm}$ artillery projectiles. J Def Anal Logist. Vol. 3 No. 2, pp. 171-192.

[2] Kroft, L. (2016). The Influence of the Finishing Strategy on the Quality of the Surface, Proceedings of the 27th DAAAM International Symposium, pp.0524-0533, B. Katalinic (Ed.), Published by DAAAM International, ISBN 978-3-902734-08-2, Vienna, Austria.

[3] Mihailescu, C.; Radulescu, M. \& Coman, F. (2011). The analysis of dispersion for trajectories of fireextinguishing rocket. Recent Adv Fluid Mech Heat Mass Transf - Proc 9th IASME / WSEAS Int Conf Fluid Mech Aerodyn FMA'11, Proc 9th IASME / WSEAS Int Conf HTE’11. 2011;pp 135-40.

[4] Saghafi, F. \& Khalilidelshad, M. (2003). A Monte Carlo Dispersion Analysis of a Rocket Flight Simulation Software. Esm 2003 17Th Eur Simul Multiconference - Found Success Model Simul.2003: 9-11 June.pp:222-8.

[5] Costello, M. \& Anderson, D. (1996). Effect of internal mass unbalance on the terminal accuracy and stability of a field artillery projectile. 21st Atmos Flight Mech Conf. 29-31 July 1996, San Diego, USA; pp 814-21.

[6] Jankovic, S.; Gallant, J. \& Celens, E. (1999). Dispersion of an artillery projectile due to its unbalance. In: 18th International Symposium on Ballistics. San Antonio, USA, 15-19 November 1999. pp. 128-41.

[7] Trzun, Z.; Andric, M. \& Vrdoljak, M. (2019). Using a 3D Model to Asses Projectile's Impact Points Deviation Due to Manufacturing Errors, Proceedings of the 30th DAAAM International Symposium, pp.0791-0799, B. Katalinic (Ed.), Published by DAAAM International, ISBN 978-3-902734- 22-8, Vienna, Austria

[8] Damic, V.; Cohodar, M. \& Kobilica, N. (2019). Development of Dynamic Model of Robot with Parallel Structure Based on 3D CAD Model, Proceedings of the 30th DAAAM International Symposium, pp.0155-0160, B. Katalinic (Ed.), Published by DAAAM International, ISBN 978- 3-902734-22-8, Vienna, Austria.

[9] Fresconi, F.; Guidos, B.; Celmins, I. \& Hathaway, W. (2016). Flight behavior of an asymmetric body through spark range experiments using roll-yaw resonance for yaw enhancement. AIAA Atmospheric Flight Mechanics Conference. 1996, 4-8 January, San Diego, USA.

[10] Oberle, W. (2015). Monte Carlo Simulations: Number of Iterations and Accuracy. US Army Res Lab. 